that such widespread use of antimicrobial treatment induces among the vulnerable inhabitants of neonatal units (whether treated or not) may contribute to their problems.

Meanwhile, Pichichero and Todd ${ }^{29}$ have shown that $96 \%$ of carefully assessed bacteraemic cultures are positive after 48 hours of incubation and $98 \%$ after 72 hours. When the culture remains negative after two to three days the risk of stopping antimicrobial treatment is therefore slight; prompt abandonment of unnecessary treatment could perhaps lessen alteration of host flora and the emergence of resistant organisms. No arbitrary rule can be laid down for length of treatment when cultures are positive: both gestational age and the presence or absence of meningitis have to be taken into account.

The combination of penicillin and an aminoglycoside such as gentamicin should meet most bacterial eventualities; higher penicillin blood concentrations are, however, needed to deter group B beta-haemolytic streptococci (at present the most common cause of early bacteraemia) than other susceptible organisms. ${ }^{30}$ The importance of anaerobic sepsis has been underestimated in the past, but as yet there are few reports of the use of metronidazole in the newborn. Though there is no proof of its efficacy by controlled trials, exchange transfusion with fresh blood may boost the infant's immune defences. ${ }^{31-33}$ Ventilatory support may be needed for some severely ill infants. Other complications that should be borne in mind are both hypoglycaemia and hyponatraemia. Finally, previously unsuspected underlying diseases ${ }^{34}$ must be considered if the infant does not improve when infection is controlled.

1 Silverman, W A, and Homan, W E, Pediatrics, 1949, 3, 157.

2 Klein, J O, and Marcy, S M, in Infectious Diseases of the Fetus and Newborn Infant, ed J S Remington and J O Klein, p 747. Philadelphia, London, Toronto, W B Saunders, 1976.

3 Bergqvist, G, Eriksson, M, and Zetterström, R, Acta Paediatrica Scandinavica, 1979, 68, 337.

${ }^{4}$ Buetow, K C, Klein, S W, and Lane, R B, American fournal of Diseases of Children, 1965, 110, 29.

5 Ablow, R C, et al, New England fournal of Medicine, 1976, 294, 65.

6 Jeffery, H, et al, Archives of Disease in Childhood, 1977, 52, 683.

7 Cowett, $\mathrm{R}$ M, et al, fournal of Pediatrics, 1976, 88, 1035.

${ }^{8}$ Mangurten, H H, and LeBeau, L J, Fournal of Pediatrics, 1977, 90, 990.

${ }^{9}$ Daum, R S, and Smith, A L, Clinical Obstetrics and Gynecology, 1979, 22 385

10 Gregory, J, and Hey, E, Archives of Disease in Childhood, 1972, 47, 747.

11 Zipursky, A, et al, Pediatrics, 1976, 57, 839.

12 Boyle, R J, et al, Pediatrics, 1978, 62, 744.

13 Manroe, B L, et al, fournal of Pediatrics, 1977, 91, 632

${ }^{14}$ Faden, H S, fournal of Pediatrics, 1976, 88, 1032.

15 Slack, M P E, and Mayon-White, R T, Archives of Disease in Childhood, $1978,53,540$

16 Ramos, A, and Stern, L, American fournal of Obstetrics and Gynecology, $1969,105,1247$.

17 Scanlon, J, fournal of Pediatrics, 1971, 79, 247.

18 Overbach, A M, Daniel, S J, and Cassady, G, Fournal of Pediatrics, 1970 76, 22.

${ }^{19}$ Adler, S M, and Denton, R L, fournal of Pediatrics, 1975, 86, 942.

20 Philip, A G S, and Hewitt, J, Pediatric Research, 1978, 12, 497 (abstract 802).

${ }^{21}$ Ehrenkranz, R A, Shephard, J, and Gross, I, Pediatric Research, 1979, 13, 459 (abstract 802).

22 Edwards, M S, and Baker, C J, Fournal of Pediatrics, 1979, 94, 286.

${ }^{23}$ Cummins, M C, unpublished data.

${ }^{24}$ Hutter, J J, Hathaway, W E, and Wayne, E R, fournal of Pediatrics, 1976, 88, 1026.

25 Xanthou, M, Archives of Disease in Childhood, 1970, 45, 242.

${ }_{26}$ Manroe, B L, et al, Fournal of Pediatrics, 1979, 95, 89.

27 Coulombel, L, et al, Acta Paediatrica Scandinavica, 1979, 68, 709.

${ }^{28}$ Hammerschlag, M R, et al, New England fournal of Medicine, 1977, 296, 1268.

${ }^{29}$ Pichichero, M E, and Todd, J K, fournal of Pediatrics, 1979, 94, 958

${ }^{30}$ McCracken, G H, and Nelson, J D, Antimicrobial Therapy for Newborns. Practical Application of Pharmacology to Clinical Usage, p 10. New York, San Francisco, London, Grune and Stratton, 1977.

31 Prod'hom, L S, et al, Pediatrics, 1974, 53, 170.

32 Xanthou, M, et al, Archives of Disease in Childhood, 1975, 50, 901.

${ }^{33}$ Pelet, B, Archives of Disease in Childhood, 1979, 54, 687.

${ }^{34}$ Levy, H L, et al, New England fournal of Medicine, 1977, 297, 823.

\section{Epidemiology of multiple sclerosis}

Although both environmental and genetic factors probably contribute to multiple sclerosis, environmental causes are thought to be the more important. Epidemiologists studying multiple sclerosis are sometimes said to be working "with their hands tied behind their backs" because there is no diagnostic laboratory test for the disease, ${ }^{1}$ but in fact epidemiological research has provided most of the information on environmental factors. Much of this research has been essentially descriptive, concentrating on analysis of the prevalence, geographical distribution, and varying incidence of the disease among different populations.

Multiple sclerosis is most frequent in Western Europe, southern Canada, the northern United States, southern Australia, and New Zealand; and in these so-called highfrequency areas its prevalence runs as high as $30-80$ cases per 100000 population. In low-frequency areas such as Asia, Central America, and most of Africa the rates are fewer than five per $100000 .^{2}$ Broadly speaking, the disease is prevalent in areas with temperate climates and less common in largely tropical or subtropical and often developing countries with a lower level of hygiene and health services. In the highfrequency areas the disease seems to occur in clusters or foci; and in Scandinavia, for example, there may be a single focus extending across all three countries. ${ }^{3}$

One of the most fruitful subjects of research has been the changes in prevalence in migrants, major differences being found among people of the same genetic stock living in different environments. Such studies have been reported from South Africa, ${ }^{4}$ Israel, ${ }^{5}$ Hawaii, ${ }^{6}$ and America, ${ }^{7}$ and the findings are remarkably similar: the migrant tends to carry with him most but not all of the risk of his native land. The age of migration seems to be important. For example, someone who moves from a high- to a low-risk zone after the age of 15 has a much higher risk of later developing multiple sclerosis than someone moving before the age of 15 . This clearly implies an important environmental factor in the pathogenesis of multiple sclerosis operating in the first 15 years of life.

What this factor might be has been much debated. The possibility examined in most detail has been infection. Studies of specific immunoglobulins have shown that titres of antibodies to certain childhood infections, notably measles, ${ }^{8}$ are increased in patients with multiple sclerosis; and at least one study has shown association of the disease with childhood infectious illnesses. ${ }^{9}$ Further support for an infectious cause for multiple sclerosis has come from a recent epidemiological study in the Faroe islands. ${ }^{10}$ From 1920 to 1977 a total of 25 cases of multiple sclerosis occurred among native-born resident Faroese and in 24 of these the onset of disease was between 1943 to 1960 . These 24 cases are said to meet all the criteria for a point-source epidemic, and-at least in the Faroes -multiple sclerosis may prove to be a transmissible disease.

Unfortunately, one of the problems of epidemiological studies in multiple sclerosis is that the picture has become even more confused as more information has become available. For example, in 1971 an association was suggested between the disease and dental caries, possibly with dental trauma and its accompanying local anaesthesia as a precipitating factor. ${ }^{11} \mathrm{~A}$ recent study ${ }^{12}$ has explored this association further and shown that death rates due to multiple sclerosis in Australia and American do show a positive correlation with dental caries. 
Furthermore, these studies have suggested that the prevalence of multiple sclerosis appears to correlate with the percentage of edentulous individuals in certain countries.

The relevance of these findings to the pathogenesis of multiple sclerosis is uncertain, but only by pursuing such investigations will we eventually unlock the key to this common and disabling disease. Sceptics might remember that epidemiological research has largely provided the evidence for the most widely accepted theory: namely, that in genetically susceptible individuals the disease follows a childhood infection with an as yet undetermined virus that remains dormant for many years before initiating, either directly or by an immunological mechanism, the onset of symptoms. ${ }^{1314}$

1 Acheson, E D, British Medical Bulletin, 1977, 33, 9.

2 Kurtzke, J F, Fournal of Neurology, 1977, 215, 1, 226.

3 Kurtzke, J F, Internal Archives of Allergy, 1969, suppl 1, 36, 59.

4 Dean, G, and Kurtzke, J F, British Medical fournal, 1971, 3, 725.

5 Leibowitz, U, Kahana, E, and Alter, M, Lancet, 1969, 2, 1323.

${ }^{6}$ Alter, M, and Okihiro, M, Neurology, 1971, 21, 1030

${ }^{7}$ Kurtzke, J F, Beebe, G W, and Norman, J E, Neurology, 1979, 29, 579.

8 Adams, J M, and Imagawa, D T, Proceedings of the Society for Experimental Biology and Medicine, 1962, 111, 562.

9 Alter, M, and Cendrowski, W, Neurology, 1976, 26, 201.

10 Kurtzke, J F, and Hyllested, K, Annals of Neurology, 1979, 5, 6.

11 Currier, R D, and Jackson, M, in Proceedings of the 96th Neurological Association, p 67. Washington, American Neurological Association 1971.

${ }^{12}$ Craelius, W, Fournal of Epidemiology and Community Health, 1978, 32, 155.

${ }^{13}$ Batchelor, J R, Compston, A, and McDonald, W I, British Medical Bulletin, 1978, 34, 279.

14 Millar, J H D, Multiple Sclerosis: A Disease Acquired in Childhood. Springfield, Illinois, Thomas, 1971.

\section{Hypertensive encephalopathy}

The term hypertensive encephalopathy was coined by Oppenheimer and Fishberg in 1928 in the case of a patient with acute nephritis complicated by cerebral symptoms and severe hypertension. ${ }^{1}$ It is a descriptive term rather than a precise pathological entity, and it has come to mean the largely reversible cerebral disorders associated with high blood pressure in the absence of evidence of cerebral thrombosis or haemorrhage. $^{2}$ Hypertensive encephalopathy occurs most frequently with acute nephritis, eclampsia, and malignant hypertension, though it may complicate the course of "benign" hypertension. ${ }^{3}$ The common factor in these diverse conditions is a rapid and sustained rise in blood pressure, and one reason for the increasing rarity of hypertensive encephalopathy is that effective drugs are now available for hypertension in general and in pregnancy in particular.

The most attractive hypothesis to explain the cerebrovascular lesions is failure of the normal autoregulatory vasoconstriction of the cerebral arterioles which accompanies a given rise in pressure. ${ }^{4}$ Sudden large increases in cerebral blood flow have been observed when the blood pressure has been raised experimentally. This "autoregulatory breakthrough" might be expected to damage the cerebral arterioles and so account for the clinical and pathological features of hypertensive encephalopathy. ${ }^{5}$

The most prominent symptoms are headache and disturbances of consciousness, while generalised and focal seizures are uncommon. ${ }^{6}$ Nausea, vomiting, and visual disturbances may also occur, and there may be focal neurological deficits. Differentiation from cerebral infarction or subarachnoid haemorrhage can be difficult in such cases.

The crucial decision in the management of patients with hypertensive encephalopathy is whether the blood pressure should be lowered within minutes or hours and by how much. The height of the blood pressure should not be the sole determining factor. None the less, except where real doubt exists as to the diagnosis, the pressure should be reduced promptly by intravenous drugs. The aim should be a controlled reduction to a diastolic pressure of around $100 \mathrm{~mm} \mathrm{Hg}$, at least initially (except in younger patients with acute nephritis or eclampsia in whom the blood pressure was presumably previously normal). Further drugs may then be given by mouth to reduce the pressure to normotensive levels over the next few days. Excessive reduction of blood pressure in hypertensive patients may be dangerous, since the lower limit of the autoregulatory threshold for cerebral blood flow may be set higher than in normotensive subjects, ${ }^{7}$ and permanent blindness has been reported in patients treated urgently for severe hypertension. ${ }^{8} 9$ Thus the initial lowering of blood pressure should usually not be complete, and a drug should be chosen that allows a predictable and controlled reduction in pressure. The drug of choice is probably sodium nitroprusside, which directly relaxes arteriolar and venular smooth muscle, the increase in venous capacitance reducing venous return to the heart so that despite a modest increase in heart rate the cardiac output is unchanged. ${ }^{10}$ Nitroprusside should be administered by infusion pump and the rate adjusted according to the response. An incremental infusion rate in the dose range $0.5-10 \mu \mathrm{g} / \mathrm{kg} / \mathrm{min}$ should control the pressure without the accumulation of cyanide (to which nitroprusside is broken down before its further metabolism to thiocyanate in the liver). The close observation of patients on this treatment requires special nursing and intensive care facilities.

Diazoxide is a more familiar drug, but less satisfactory because the very nature of its administration in a rapid intravenous bolus exposes patients to the risk of an excessive fall in pressure, especially in those already having antihypertensive treatment or those who have renal failure. ${ }^{11}$ To minimise this risk a useful and probably safer regimen would be more frequent administration of small bolus injections of $100 \mathrm{mg}$ repeated every 5-10 minutes until the pressure has fallen adequately.

Treatment with a beta-blocker by mouth should be started at this point, and further control of the blood pressure may be maintained by the addition of oral hydrallazine. Treatment with a diuretic will almost certainly be required too, and if there is impairment of renal function a potent loop diuretic such as frusemide or bumetanide will be needed. Should generalised convulsions occur they should be treated with intravenous diazepam and this may be necessary before intravenous hypotensive therapy can be started.

Patients with hypertensive encephalopathy need to have very careful follow-up with respect to the control of their blood pressure, especially those patients who also have malignant hypertension. In a recent study of the quality of care delivered to patients with malignant hypertension who subsequently died, ${ }^{12}$ a major finding was the poor control of blood pressure after diagnosis. Only $27 \%$ of the patients had an average treated blood pressure of less than $110 \mathrm{~mm} \mathrm{Hg}$. There is now little doubt that even the most refractory patients will respond on a long-term basis to drugs-provided their compliance with treatment is satisfactory. If initial treatment with a combination of propranolol, hydrallazine, and a diuretic does not lower the blood pressure to normal then the more potent vasodilator minoxidil will usually be successful. ${ }^{13}$ 